\title{
MiR-34c induces apoptosis and inhibits the viability of M4e cells by targeting BCL2
}

\author{
RUI LI $^{1 *}$, HONGXIA ZHANG ${ }^{2 *}$ and XILING ZHENG ${ }^{3}$ \\ ${ }^{1}$ Department of Otorhinolaryngology, Hospital of Laiwu Iron and Steel Group Company, Laiwu, Shandong 271100; \\ ${ }^{2}$ Department of ENT, Hanzhong 3201 Hospital Affiliated to Xi'an Jiaotong University of Medicine, Hanzhong, Shaanxi 723000; \\ ${ }^{3}$ Department of Otorhinolaryngology, Yanan University Affiliated Hospital, Yanan, Shaanxi 716000, P.R. China
}

Received July 26, 2016; Accepted November 2, 2017

DOI: $10.3892 / \mathrm{ol} .2017 .7640$

\begin{abstract}
The present study aimed to investigate microRNA (miR/miRNA)-34c expression and the association of miR-34c with B cell lymphoma 2 (BCL2) in M4e laryngeal carcinoma cell line. M4e laryngeal carcinoma cells were cultured and transfected with lenti-miR-34c or scramble miRNA for $72 \mathrm{~h}$. Cell viability and the percentage of cells undergoing apoptosis of transfected cells were detected using MTT and Annexin V/allophycocyanin and propidium iodide assays, respectively. Reverse transcription-quantitative polymerase chain reaction (RT-qPCR) and western blot analysis were performed to determine BCL2 mRNA and protein expression in transfected M4e cells. In addition, luciferase reporter assay was performed to identify whether BCL2 is a direct target of miR-34c. Transfection of lenti-miR-34c was able to significantly inhibit cell viability $(\mathrm{P}<0.01)$, increase the percentage of cells undergoing apoptosis $(\mathrm{P}<0.001)$ and downregulate BCL2 protein expression $(\mathrm{P}<0.01)$ in M4e cells. $\mathrm{RT}-\mathrm{qPCR}$ data revealed that lenti-miR-34c transfection did not affect BCL2 mRNA expression. However, data from the luciferase reporter assay revealed that transfection with $\mathrm{miR}-34 \mathrm{c}$ negative control decreased luciferase activity in M4e cells co-transfected with pGL3-BCL2-MUT plasmid, compared with miR-34c inhibitor $(\mathrm{P}<0.01)$. Collectively, the results from the present study provided evidence that miR-34c may be involved in the pathogenesis of laryngeal cancer, and BCL2 may be negatively regulated by $\mathrm{miR}-34 \mathrm{c}$ in $\mathrm{M} 4 \mathrm{e}$ cells.
\end{abstract}

Correspondence to: Miss Xiling Zheng, Department of Otorhinolaryngology, Yanan University Affiliated Hospital, 43 Beida Street, Baota, Yanan, Shaanxi 716000, P.R. China

E-mail: cyi8f5@sina.com

*Contributed equally

Abbreviation: BCL2, B cell lymphoma 2

Key words: microRNA-34c, B cell lymphoma 2, M4e cells, apoptosis

\section{Introduction}

Laryngeal cancer is a malignant cancer commonly occurring in the head and neck region, and was ranked as the sixth most frequent cancer worldwide in $2004(1,2)$. However to date, the treatment strategy for laryngeal cancer is primarily surgery with radiotherapy. Therefore, there is an urgent requirement to investigate the causes and treatment strategies to further improve the survival of patients.

A number of protein-coding and non-coding genetic factors have been revealed to be abnormally expressed in laryngeal cancer samples or cell lines (1,3-5). Correspondingly, two genes and microRNAs (miRNA/miRs) are considered to be key biological factors in laryngeal cancer, including SOX2 (5), glucose transporter 1/3 (6), miR-196a (7), miR-221 (8) and miR-34a/c (2). miRNAs are small noncoding RNA molecules, which generally negatively regulate gene expression at the post-transcriptional level. An increasing number of studies indicate that ectopic expression of miRNAs is associated with cancer pathogenesis. For example, aberrant miR-221 expression was reported to accelerate the proliferation of M4e laryngeal carcinoma cell line by suppressing apoptotic protease activating factor 1 (Apaf-1) (8). Inhibition of miR-221 in vitro increased the expression levels of Apaf-1 apoptotic pathway proteins, including caspase-3 and -9 (8). Furthermore, miR-34a/c expression was observed to be downregulated in human laryngeal squamous cell carcinoma tissues, and upregulation of miR-34a/c in Hep-2 cells by transfection significantly decreased cell proliferation and migratory ability in vitro (2). It was previously reported that B cell lymphoma 2 (BCL2), a central genetic apoptotic regulator (9), which is crucial for cell apoptosis, migration and invasion (3), is involved in the pathogenesis of laryngeal cancer (10-12). In addition, research into other diseases has reported on the association between miR-34c and BCL2 (13-15); there is an inverse association between levels of miR-34c and expression of BCL2. Knockdown of miR-34c upregulated anti-apoptotic protein BCL2 to ameliorate apoptosis in the hippocampus or promote embryonic stem cell (ESC) differentiation (13-15). Based on these reports, the authors of the present study hypothesized that there may be an association between miR-34c and BCL2, which is involved in the pathogenesis of laryngeal cancer. However, to the best our knowledge, to date, there have been no reports of direct 
evidence for the association between miR-34c and BCL2 in laryngeal cancer.

In order to investigate the association between miR-34c and BCL2 in laryngeal cancer, miR-34c was overexpressed to investigate the effect of miR-34c overexpression on viability, apoptosis and BCL2 expression in M4e cells. In addition, a luciferase reporter assay was employed to confirm whether BCL2 is a direct target of miR-34c. The aim of the present study was to provide further information on whether BCL2 may be regulated by miR-34c and to investigate if there is any therapeutic target potential of miR-34c in M4e cells.

\section{Materials and methods}

Cell lines and culture conditions. Culture of M4e cells was performed as reported previously by Wang et al (16). Human laryngeal cancer cells M4e were purchased from the American Type Culture Collection (Manassas, VA USA). Briefly, the cells were cultured in Dulbecco's modified Eagle's medium (DMEM; Gibco; Thermo Fisher Scientific, Inc., Waltham, MA, USA) supplemented with $10 \%$ fetal bovine serum (FBS, Thermo Fisher Scientific, Inc.), $100 \mathrm{U} / \mathrm{ml}$ penicillin and $100 \mu \mathrm{g} / \mathrm{ml}$ streptomycin at $37^{\circ} \mathrm{C}$ in a humidified atmosphere with $5 \% \mathrm{CO}_{2}$ and $95 \%$ air.

Lentiviral vector construction and cell transfection. The transfection of M4e cells was performed via Lipofectamine $2000^{\circledR}$ reagent (Invitrogen; Thermo Fisher Scientific, Inc.). Then, the cells were divided into three groups: Control group (control), scrambled group (scramble), and has-miR-34c transfected group (lenti-miR-34c). Total RNA in M4e cells was extracted by using the TRIzol reagent (Invitrogen; Thermo Fisher Scientific, Inc.). As previously reported by Yang et al (17), the full length pre-miR-34c oligonucleotide was synthesized by Shanghai GeneChem Co., Ltd. (Shanghai, China) and was subsequently inserted into the unique EcoRI site of the GV217 lentiviral vector (Shanghai GeneChem Co., Ltd.), to construct a lentivirus encoding miR-34c, which is named lenti-miR-34c. The nucleotide sequence of the construct was then confirmed by sequencing. In accordance with Yang et al (17), the complementary nucleotides of miR-34c were cloned at one site between the AgeI and EcoRI sites in GV280 lentiviral vector (Shanghai GeneChem Co., Ltd.) to construct the specific inhibitor of miR-34c. M4e cells were seeded in 6-well plates at a density of $5 \times 10^{4}$ cells/well and cultured in DMEM media containing $10 \% \mathrm{FBS}, 100 \mathrm{U} / \mathrm{ml}$ penicillin and $100 \mu \mathrm{g} / \mathrm{ml}$ streptomycin at $37^{\circ} \mathrm{C}$ with $5 \% \mathrm{CO}_{2}$. When $30 \%$ confluence was obtained, the cells were transfected with lenti-miR-34c or scramble for $72 \mathrm{~h}$. The efficiency of transfection was evaluated by EGFP fluorescence intensity on a Nikon Eclipse 80i microscope (Nikon USA, Melville, NY, USA).

Cell viability assay. MTT assay (Sigma-Aldrich; Merck KGaA, Darmstadt, Germany) was used to determine the relative cell viability of infected M4e cells at 24, 48, 72 and $96 \mathrm{~h}$ post-transfection (2). Control and infected M4e cells by lenti-miR-34c were seeded in 96-well plates at a density of $1 \times 10^{3}$ cells/well. M4e cells were incubated in $100 \mu 1$ DMEM with $10 \mu \mathrm{l} \mathrm{MTT}$ solution at $37^{\circ} \mathrm{C}$ for $2 \mathrm{~h}$. The optical density was determined at an absorbance wavelength of $570 \mathrm{~nm}$. Each experiment was performed in triplicate.

Apoptosis assay. Cell apoptosis was determined using the Annexin V apoptosis detection kit (KeyGen Biotech, Nanjing, China), as previously described (4). In brief, the cells were washed twice with phosphate-buffered saline (PBS), resuspended in $1 \mathrm{x}$ binding buffer (BD Biosciences, San Jose, CA, USA) and dual staining was performed with Annexin V/allophycocyanin (APC) and propidium iodide (PI) for $10 \mathrm{~min}$ at the room temperature in darkness, according to the manufacturer's protocol. M4e cells that were untreated were used as the internal control. Each experiment was repeated three times. The percentage of M4e cells undergoing apoptosis was determined by flow cytometric analysis using a flow cytometer (FACSCalibur; BD Biosciences, San Jose, CA, USA) and FlowJo Software (version 10.4; FlowJo LLC, Ashland, OR, USA).

Reverse transcription-quantitative polymerase chain reaction (RT- $q P C R)$. For miR-21 and BCL2 mRNA analyses, total RNA was extracted from M4e cells using TRIzol reagent (Invitrogen; Thermo Fisher Scientific, Inc.), according to the manufacturer's protocol. Then, $4 \mu \mathrm{g}$ of total RNA was converted to cDNA using PrimeScript II 1st Strand cDNA Synthesis kit (Takara Bio Inc., Otsu, Japan) according to the manufacturer's protocol. For the detection of miR-21 expression, stem-loop RT-qPCR was performed using SYBR Premix Ex Taq ${ }^{\mathrm{TM}}$ kit (Takara Bio Inc., Otsu, Japan) and a LightCycler 480 instrument (Roche Diagnostics, Basel, Switzerland) equipped with Light-Cycler Software Version 1.5 (Roche Diagnostics) following the manufacturer's instructions. The PCR cycling consisted of $95^{\circ} \mathrm{C}$ for $5 \mathrm{~min}$, followed by 40 cycles of $95^{\circ} \mathrm{C}$ for $30 \mathrm{sec}, 57^{\circ} \mathrm{C}$ for $30 \mathrm{sec}$ and $72^{\circ} \mathrm{C}$ for $30 \mathrm{sec}$. U6 small RNA was used as the reference RNA to normalize relative expression of miR-21. BCL2 mRNA expression was detected via RT-qPCR using QuantiTect SYBR Green RT-PCR kit (Qiagen GmbH, Hilden, Germany). $\beta$-actin was used to normalize the relative expression level of BCL2 mRNA. . Fold changes were calculated using the $2^{-\Delta \Delta C t}$ method (5). The sequences of RT-qPCR primers were as follow: miR-21, Stem-loop RT-qPCR primer: 5'-GTCGT ATCCAGTGCAGGGTCCGAGGTATTCGCACTGGATAC GACTCAACA-3', Forward: 5'-GCCCGCTAGCTTATCAGA CTGATG-3', Reverse: 5'-GTGCAGGGTCCGAGGT-3'; U6, RT primer: 5'-GTCGTATCCAGTGCAGGGTCCGAGGTA TTCGCACTGGATACGACAAAAATATG-3', Forward: 5'-GCGCGTCGTGAAGCGTTC-3', Reverse: 5'-GTGCAG GGTCCGAGGT-3'; BCL2 mRNA, Forward: 5'-CTGTGC TGCTATCCTGC-3', Reverse: 5'-TGCAGCCACAATACT GT-3'; $\beta$-actin, Forward: 5'-TCACCCACACTGTGCCCC ATCTACGA-3', Reverse: 5'-CAGCGGAACCGCTCATTG CCAATGG-3'.

Western blot analysis. Western blot analysis was performed as previously described by Li et al (3). M4e cells were harvested after being digested with $0.25 \%$ trypsin (Thermo Fisher Scientific, Inc.) for $2 \mathrm{~min}$ at $37^{\circ} \mathrm{C}$, washed in PBS and resuspended in radioimmunoprecipitation assay cell lysis solution (Beyotime Institute of Biotechnology, Shanghai, 
China) for $30 \mathrm{~min}$ at $4^{\circ} \mathrm{C}$. Protein concentration was determined using the bicinchoninic acid assay (Pierce; Thermo Fisher Scientific, Inc.). Cell lysates with equal amounts of protein $(20 \mu \mathrm{g} /$ well) were separated on $10 \%$ SDS-PAGE gels by electrophoresis, and transferred to polyvinylidene difluoride (PVDF) membranes (Invitrogen; Thermo Fisher Scientific, Inc.). PVDF membranes were blocked with 5\% skimmed milk in TBST at room temperature with agitation for $1 \mathrm{~h}$. The PVDF membranes were incubated with the primary antibody against BCL2 (ab59348; dilution, 1:1,000; Epitomics; Abcam, Cambridge, UK) at $4^{\circ} \mathrm{C}$ for $12 \mathrm{~h}$. The membranes were then washed three times with TBST each for $10 \mathrm{~min}$ and incubated with goat anti-rabbit immunoglobulin $\mathrm{G}$ antibodies (ab7090; dilution, 1:1,000; Epitomics; Abcam) at room temperature for $1 \mathrm{~h}$. The blots were washed with TBST, and then polypeptide bands were detected using ECL Plus Western Blotting Detection Reagents (Merck KGaA, Darmstadt, Germany) and were exposed to X-ray film (Kodak, Rochester, NY, USA). The intensity of the bands was quantified using Image $\mathrm{Lab}^{\mathrm{TM}}$ Software (Bio-Rad Laboratories, Inc., Hercules, CA, USA). $\beta$-actin (ab8227; dilution, 1:1,000; Epitomics; Abcam) was used as an internal reference control.

Luciferase reporter assay. A luciferase reporter assay was performed, according to the methods provided by Li et al (3). In brief, M4e cells were seeded and cultured in 6-well plates at a density of $5 \times 10^{4}$ cells/well. Upon reaching $80 \%$ confluence, the cells were transfected with either pGL3-BCL2-WT (wildtype) or pGL3-BCL2-MUT (mutant) vector containing firefly luciferase together with miR-34c inhibitor or negative control. M4e cells were transfected with WT or mutant reporter plasmid using Lipofectamine $200{ }^{\circledR}$ for $6 \mathrm{~h}$, and were subsequently co-transfected with miR-34c inhibitor or negative control for $36 \mathrm{~h}$. The dual luciferase assay system (Promega Corporation, Madison, WI, USA) was used to measure luciferase activity, according to the manufacturer's protocol.

Statistical analysis. All data are expressed as the mean \pm standard deviation of three independent experiments. The differences between two groups were assessed using an unpaired, two-tailed Student's t-test, and multi-group comparisons were analyzed by one-way analysis of variance with Bonferroni analyses performed as post-hoc tests. $\mathrm{P}<0.05$ was considered to indicate a statistically significant difference.

\section{Results}

miR-34c inhibits the viability of M4e cells in vitro. The viability of M4e cells was assessed by MTT assay. As indicated in Fig. 1, the viability of M4e cells was inhibited following transfection with lenti-miR-34c for $72 \mathrm{~h}$. Cell viability in the control and scramble groups gradually increased and reached the highest level at $96 \mathrm{~h}$ post-transfection. By contrast, the cell viability in lenti-miR-34c transfected-group gradually decreased to the lowest level at $96 \mathrm{~h}$ post-transfection. Significant differences in cell viability were determined between the control or scramble group and lenti-miR-34c group at 48, 72 and $96 \mathrm{~h}$ post-transfection $(\mathrm{P}<0.01$ or $\mathrm{P}<0.001)$.

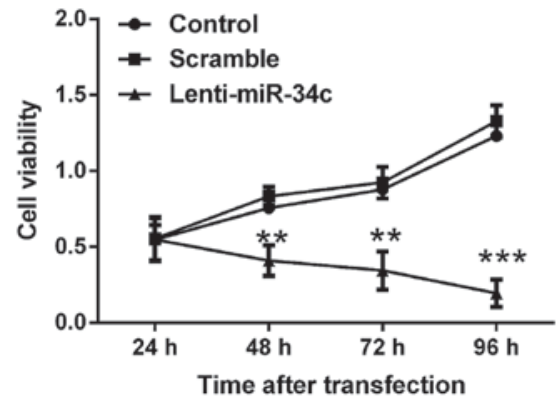

Figure 1. Effect of miR-34c on the viability of M4e cells. The cells were infected with lenti-miR-34c or scramble for $72 \mathrm{~h}$. The efficiency of infection was evaluated by EGFP fluorescence intensity at 24, 48, 72 and $96 \mathrm{~h}$ post-transfection. All data are expressed as the mean \pm standard deviation (SD) of three independent experiments. ${ }^{* *} \mathrm{P}<0.01,{ }^{* * * *} \mathrm{P}<0.001$ vs. control and scramble. miR, microRNA.

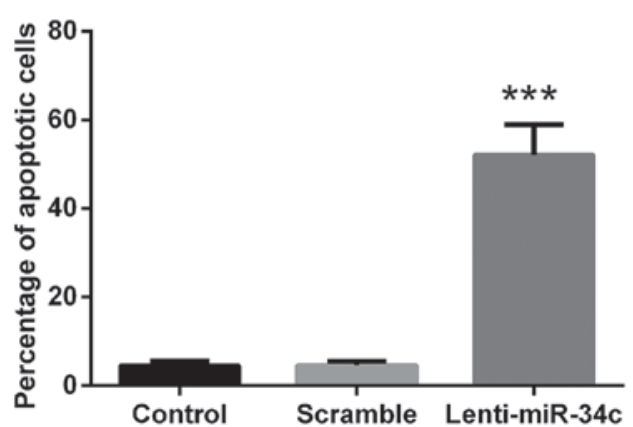

Figure 2. Effect of miR-34c on the apoptosis of M4e cells. The cells were infected with lenti-miR-34c or scramble for $72 \mathrm{~h}$. M4e cells were stained with Annexin V/allophycocyanin and propidium iodide. The percentage of cells undergoing apoptosis was determined by flow cytometric analysis. All data are expressed as the mean \pm standard deviation of three independent experiments. ${ }^{* * *} \mathrm{P}<0.001$ vs. control.

miR-34c induces the apoptosis of M4e cells in vitro. M4e cells were stained with Annexin V/APC and PI to evaluate the percentage of cells undergoing apoptosis in the three treatment groups. The results indicated that lenti-miR-34c transfection was able to promote the apoptosis of M4e cells, where the percentage of apoptotic cells in the lenti-miR-34c group was $\sim 65 \%$ and the percentage in the control groups was $6 \%$ $(\mathrm{P}<0.001$; Fig. 2).

Effect of miR-34c on $\mathrm{mRNA}$ and protein levels of BCL2. To investigate the effect of lenti-miR-34c transfection on BCL2 expression in M4e cells, RT-qPCR and western blot analyses were performed to analyze the levels of mRNA and protein, respectively. RT-qPCR results indicated that BCL2 mRNA expression did not change following lenti-miR-34c transfection (Fig. 3A). However, western blot analysis revealed that the level of BCL2 protein was significantly downregulated by lenti-miR-34c transfection compared with the control and scramble groups $(\mathrm{P}<0.01$; Fig. $3 \mathrm{~B}, \mathrm{C})$. This indicated that BCL2 may be a putative target of miR-34c.

miR-34c modulates BCL2 expression by targeting BCL2. To verify whether BCL2 was a putative target of miR-34c, M4e cells were co-transfected with either pGL3-BCL2-WT or pGL3-BCL2-MUT vector containing firefly luciferase 

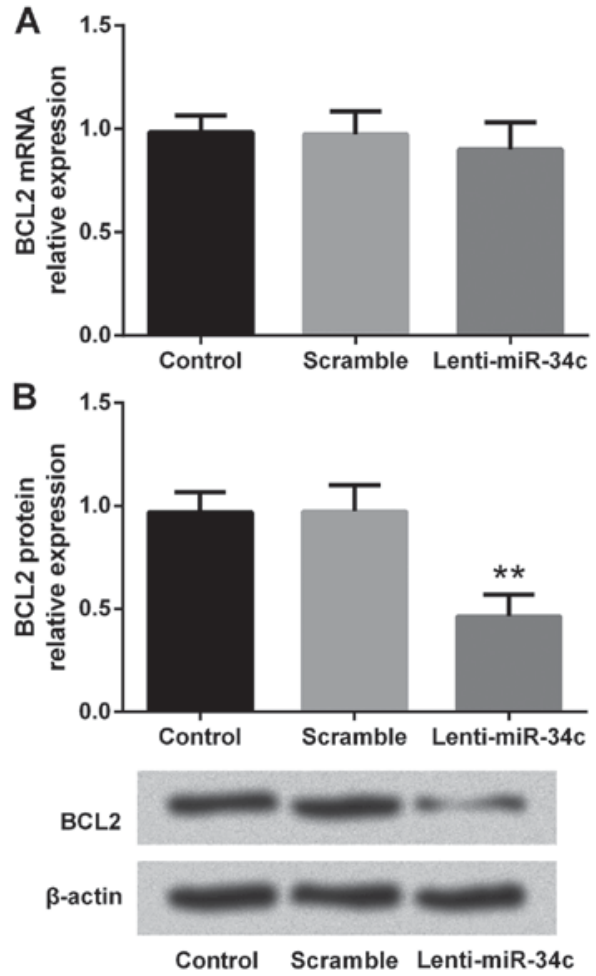

Figure 3. Effect of miR-34c on BCL2 mRNA and protein expression Analyses by Reverse transcription-quantitative polymerase chain reaction and western blotting were employed to investigate the (A) mRNA and (B) protein expression level of BCL2, respectively. All data are expressed as the mean \pm standard deviation of three independent experiments. The cells were infected with lenti-miR-34c or scramble for $72 \mathrm{~h} .{ }^{* *} \mathrm{P}<0.01$ vs. control and scramble. BCL2, B cell lymphoma 2.

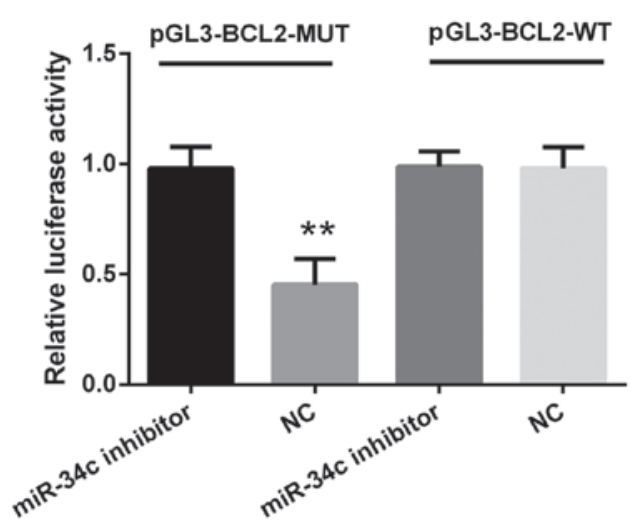

Figure 4. Levels of wildtype or mutant BCL2 in M4e cells in the presence of miR-34c inhibitor or negative control. M4e cells were co-transfected either pGL3-BCL2-WT or pGL3-BCL2-MUT vector containing firefly luciferase using Lipofectamine $2000^{\circledR}$ for $6 \mathrm{~h}$ and were then subsequently transfected again with miR-34c inhibitor or NC for $36 \mathrm{~h}$. Luciferase activity was measured using a dual luciferase assay system. ${ }^{* *} \mathrm{P}<0.01 \mathrm{vs}$. miR-34c inhibitor. WT, wildtype; MUT, mutant; NC, negative control.

together with miR-34c inhibitor or negative control. As indicated in Fig. 4, luciferase activity in M4e cells co-transfected with miR-34c negative control and pGL3-BCL2-MUT was decreased compared with the miR-34c inhibitor $(\mathrm{P}<0.01)$. In addition, no significant differences were observed between cells transfected with WT plasmids when co-transfected with miR-34c inhibitor. The data presented in this present study indicated that BCL2 may be directly regulated by miR-34c.

\section{Discussion}

Recent studies in cancer research have revealed that hsa-miR-34c is downregulated in human laryngeal squamous cell carcinoma tissues, and ectopic expression of miR-34a/c significantly induces the proliferative and migratory capacities of Hep-2 cells, in vitro (2). The imbalance between cell proliferation and apoptosis is a phenotypic characteristic of cancer, which is largely accountable for the pathogenesis of this disease. In the present study, the results indicated that infection with lenti-miR-34c was able to significant induce apoptosis and inhibit proliferation of M4e cells. Furthermore, it was revealed that BCL2 may a putative target of miR-34c.

Over the past decades, numerous miRNAs and genes have been verified to associate with and play crucial roles in regulating biological processes, including pathopoiesis and tumorigenesis $(2,18,19)$. The target genes of these miRNAs, namely miR-34c (2), miR-196a (7), miR-221 (8), miR-203 (20), miR-27a (21) and miR-106b (22) have been reported to be associated with laryngeal carcinoma. Furthermore, multiple miRNAs have been demonstrated to exhibit tumor suppressive effects in several types of cancer (2). For example, miR-34a/c was reported to be downregulated in human laryngeal squamous cell carcinoma, and overexpression of $\mathrm{miR}-34 \mathrm{a} / \mathrm{c}$ in Hep-2 cells by transfection, decreased the proliferative and migratory abilities of the cells (2). In Li et al (2), RT-qPCR analysis indicated that UDP-N-acetyl- $\alpha$-D-galactosamine: polypeptide-N-acetylgalactosaminyl transferase 7 (GALNT7) expression was negatively regulated by miR-34a/c in Hep-2 cells. Further data from the luciferase reporter assay determined that GALNT7 was a target of miR-34a/c. These results highlighted that miR-34a/c, and its novel target GALNT7 may serve as novel potential biomarkers for human laryngeal squamous cell carcinoma therapy.

Another miRNA of interest, miR-27a, has been reported to be an important regulator in laryngeal carcinoma (21). As previously reported by Tian et al (21), miR-27a and polo-like kinase 2 (PLK2), are critically involved in controlling cell cycle progression. In addition, the expression level of miR-27a was significantly upregulated in laryngeal carcinoma tissues compared with adjacent non-tumor tissues, respectively. Transfection of miR-27a mimics increased proliferation and colony formation and decreased the percentage of cells undergoing apoptosis and the level of PLK2 protein expression in Hep-2 cells. These data suggested that miR-27a acts as an oncogene in laryngeal squamous cell carcinoma via regulating PLK2 protein and implicated miR-27a to be a potential biomarker in the diagnosis and therapy for laryngeal carcinoma.

BCL2 contributes a crucial role in cell death, apoptosis, migration and invasion $(3,9,23,24)$. Previous studies have reported that BCL2 is involved in the pathogenesis of laryngeal cancer (10-12). In the present study, it was observed that transfection of M4e cells with lenti-miR-34c significantly inhibited cell viability and BCL-2 protein expression, and increased the percentage of cells undergoing apoptosis. Furthermore, luciferase reporter assay revealed that transfection of miR-34c negative control decreased luciferase activity in M4e cells 
co-transfected with pGL3-BCL2-MUT plasmid, compared with the miR-34c inhibitor.

In conclusion, the results from the present study demonstrated that miR-34c was a growth inhibitor of M4e cells, and there was a negative association between miR-34c and its target BCL2. Downregulated miR-34c may contribute to the malignancy of human laryngeal carcinoma via BCL2-associated pathways. Furthermore, miR-34c and BCL2 may serve as biomarkers for the diagnosis and treatment of laryngeal carcinoma.

\section{References}

1. Zhang F, Xu Z, Wang K, Sun L, Liu G and Han B: microRNA and gene networks in human laryngeal cancer. Exp Ther Med 10: 2245-2252, 2015.

2. Li W, Ma H and Sun J: microRNA-34a/c function as tumor suppressors in Hep-2 laryngeal carcinoma cells and may reduce GALNT7 expression. Mol Med Rep 9: 1293-1298, 2014.

3. Li Y, Yan L, Zhang W, Wang $\mathrm{H}$, Chen W, Hu N and $\mathrm{Ou} \mathrm{H}$ miR-21 inhibitor suppresses proliferation and migration of nasopharyngeal carcinoma cells through down-regulation of BCL2 expression. Int J Clin Exp Pathol 7: 3478-3487, 2014.

4. Ren J, Zhu D, Liu M, Sun Y and Tian L: Downregulation of miR-21 modulates Ras expression to promote apoptosis and suppress invasion of Laryngeal squamous cell carcinoma. Eur J Cancer 46: 3409-3416, 2010.

5. Yang N, Hui L, Wang Y, Yang H and Jiang X: SOX2 promotes the migration and invasion of laryngeal cancer cells by induction of MMP-2 via the PI3K/Akt/mTOR pathway. Oncol Rep 31: 2651-2659, 2014

6. Starska K, Forma E, Jóźwiak P, Bryś M, Lewy-Trenda I, Brzezińska-Błaszczyk E and Krześlak A: Gene and protein expression of glucose transporter 1 and glucose transporter 3 in human laryngeal cancer-the relationship with regulatory hypoxia-inducible factor- $1 \alpha$ expression, tumor invasiveness, and patient prognosis. Tumor Biol 36: 2309-2321, 2015.

7. Saito K, Inagaki K, Kamimoto T, Ito Y, Sugita T, Nakajo S, Hirasawa A, Iwamaru A, Ishikura T, Hanaoka $\mathrm{H}$, et al: MicroRNA-196a is a putative diagnostic biomarker and therapeutic target for laryngeal cancer. PLoS One 8: e71480, 2013.

8. Sun X, Liu B, Zhao XD, Wang LY and Ji WY: MicroRNA-221 accelerates the proliferation of laryngeal cancer cell line Hep-2 by suppressing Apaf-1. Oncol Rep 33: 1221-1226, 2015.

9. Cimmino A, Calin GA, Fabbri M, Iorio MV, Ferracin M, Shimizu M, Wojcik SE, Aqeilan RI, Zupo S, Dono M, et al: miR-15 and miR-16 induce apoptosis by targeting BCL2. Proc Natl Acad Sci USA 102: 13944-13949, 2005.

10. Jin YT, Kayser S, Kemp BL, Ordonez NG, Tucker SL, Clayman GL, Goepfert H, Luna MA, Batsakis JG and El-Naggar AK: The prognostic significance of the biomarkers p21WAF1/CIP1, p53, and bcl-2 in laryngeal squamous cell carcinoma. Cancer 82: 2159-2165, 1998.
11. Du J, Chen G, Vlantis A, Chan P, Tsang R and van Hasselt C: Resistance to apoptosis of HPV 16-infected laryngeal cancer cells is associated with decreased Bak and increased Bcl-2 expression. Cancer Lett 205: 81-88, 2004

12. Gurlek U, Abakay CD, Ozkan L, Saraydaroglu O, Kurt M and Cetintas SK: The evaluation of bcl-2 expression as a prognostic marker in early stage laryngeal cancer. Tumori 99: 682-688, 2013.

13. Zirnheld AL, Regalado EL, Shetty V, Chertkow H, Schipper HM and Wang E: Target genes of circulating miR-34c as plasma protein bio markers of Alzheimer's disease and mild cognitive impairment. J Aging Sci 5: e125, 2017.

14. Cao SE, Tian J, Chen S, Zhang X and Zhang Y: Role of miR-34c in ketamine-induced neurotoxicity in neonatal mice hippocampus. Cell Biol Int 39: 164-168, 2015.

15. Zhang S, Yu M, Liu C, Wang L, Hu Y, Bai Y and Hua J: MIR-34c regulates mouse embryonic stem cells differentiation into male germ-like cells through RARg. Cell Biochem Funct 30: 623-632, 2012.

16. Wang HX and Tang C: Galangin suppresses human laryngeal carcinoma via modulation of caspase- 3 and AKT signaling pathways. Oncol Rep 38: 703-714, 2017.

17. Yang S, Li WS, Dong F, Sun HM, Wu B, Tan J, Zou WJ and Zhou DS: KITLG is a novel target of miR-34c that is associated with the inhibition of growth and invasion in colorectal cancer cells. J Cell Mol Med 18: 2092-2102, 2014.

18. Valastyan S, Reinhardt F, Benaich N, Calogrias D, Szász AM, Wang ZC, Brock JE and Richardson AL: Retraction notice to: A pleiotropically acting MicroRNA, miR-31, inhibits breast cancer metastasis. Cell 161: 417, 2015 .

19. Iorio MV and Croce CM: MicroRNA dysregulation in cancer: Diagnostics, monitoring and therapeutics. A comprehensive review. EMBO Mol Med 4: 143-159, 2012.

20. Bian K, Fan J, Zhang X, Yang XW, Zhu HY, Wang L, Sun JY, Meng YL, Cui PC, Cheng SY, et al: MicroRNA-203 leads to G1 phase cell cycle arrest in laryngeal carcinoma cells by directly targeting survivin. FEBS Lett 586: 804-809, 2012

21. Tian Y, Fu S, Qiu GB, Xu ZM, Liu N, Zhang XW, Chen S, Wang Y, Sun KL and Fu WN: MicroRNA-27a promotes proliferation and suppresses apoptosis by targeting PLK2 in laryngeal carcinoma. BMC Cancer 14: 678, 2014.

22. Ying X, Kai W, Wei G, Zhang C, Huang F, Wen S and Wang B: MicroRNA-106b regulates the tumor suppressor RUNX3 in laryngeal carcinoma cells. FEBS Lett 587: 3166-3174, 2013.

23. Kusunoki S, Kato K, Tabu K, Inagaki T, Okabe H, Kaneda H, Suga S, Terao Y, Taga T and Takeda S: The inhibitory effect of salinomycin on the proliferation, migration and invasion of human endometrial cancer stem-like cells. Gynecol Oncol 129: 598-605, 2013.

24. Yang TQ, Lu XJ, Wu TF, Ding DD, Zhao ZH, Chen GL, Xie XS, Li B, Wei YX, Guo LC, et al: MicroRNA-16 inhibits glioma cell growth and invasion through suppression of BCL2 and the nuclear factor- $\kappa$ B1/MMP9 signaling pathway. Cancer Sci 105: 265-271, 2014. 